\title{
Micro gas sensor assembly of tin oxide nano-particles by a capillary micro-molding process
}

\author{
Hiroshi FUDOUZI ${ }^{\dagger}$ and Yoshio SAKKA \\ National Institute for Materials Science, 1-2-1, Sengen, Tsukuba 305-0047
}

This paper reports a micro gas sensor assembled with tin oxide, $\mathrm{SnO}_{2}$, nano-powders. The micro gas sensors are fabricated by a modified micro molding in capillary (MIMIC) technique using an ethanol suspension with 2.0 vol\% $\mathrm{SnO}_{2}$ nano-powders. A micro pattern is formed as a set of parallel lines $23 \mu \mathrm{m}$ in width and $1.2 \mu \mathrm{m}$ in depth. The micro pattern is heat treated at $300^{\circ} \mathrm{C}$ for $5 \mathrm{~h}$. The micro-sensor was able to detect ethanol gas by measuring the electrical resistance of the tin oxide a glass tube furnace. When ethanol vapor flows through the furnace the resistance decreases and the response time is $1.9 \mathrm{~s}$. The sensor resistance ratio was determined to be 19.5 at $450^{\circ} \mathrm{C}$. Our modified MIMIC technique enables easy and simple fabrication of ethanol vapor micro-sensors in a cost-effective way.

(C2010 The Ceramic Society of Japan. All rights reserved.

Key-words : Nano-powder, Gas sensor, PDMS $\mu$-mold, Micro molding in capillaries (MIMIC), Soft-lithography, Tin oxide

[Received November 26, 2009; Accepted January 15, 2010]

\section{Introduction}

A bottom-up approach to the assembly of devices from nanopowders is a promising technology in the field of advanced ceramic processing. Recently ceramic patterns have been fabricated using the micro molding in capillary (MIMIC) process from a colloidal ceramic suspension. ${ }^{1)-3)}$ The MIMIC method was originally proposed and developed by Kim et al. ${ }^{4)}$ The mechanism of the MIMIC technique is the spontaneous capillary driven infiltration of liquid into channels of a flexible mold. The MIMIC method is a soft-lithography technique ${ }^{5), 6)}$ and therefore, is simple and easy to use and has the potential for application in a mass-production process. A flexible micro-mold consisting of a poly-dimethylsiloxane elastomer (PDMS) is fabricated by replicating a re-usable silicone master-mold. In the soft-lithography for MIMIC method, the flexible micro-mold plays an important role in micro-channel formation on a flat solid substrate.

Previous studies using the MIMIC technique have focused on the application of two main classes of starting ceramic liquids. The first class of starting liquids are sol-gel precursors, for example metal alkoxides, which are used to process oxide ceramics. ${ }^{7,8)}$ In this case, uniform patterning is observed over a wide area, because the precursor infiltrates farther into the channels of the PDMS $\mu$-molds. However, the post heat-treatment is necessary to change into a ceramic pattern of the precursor gel pattern. Thermal decomposition of the remaining organic component causes the shrinking of microstructure and affects the dimensional accuracy and integrity of the sensor.

The other class of starting liquids studied are colloidal suspensions of ceramic powders, as reported by Gauckler et al. ${ }^{1)}$ who demonstrated that low-cost ceramic microstructures are easily generated using suspensions of high solid loading (e.g., more than $15 \mathrm{vol} \%$ ). However, with colloidal suspensions, the infiltration lengths of the suspension into the capillaries is short because the suspensions are non-Newtonian fluids and therefore are con-

Corresponding author: H. Fudouzi; E-mail: FUDOUZI.Hiroshi@ nims.go.jp tinuously drying while infiltration is taking place. In order to overcome these difficulties, Moon et al. developed the vacuumassisted microfluidic lithography method ${ }^{9)-11)}$ using suspensions of high solid loading of approximately $20 \mathrm{vol} \%$. This method enabled the fabrication of defect free micro-scale patterns over an mm-scale area. In this work, we develop an alternative approach for making ceramic micro patterns at a lower solid loading ethanol suspension of a few vol\%. ${ }^{12), 13)}$

Figure 1 shows the MIMIC patterning process using suspensions of a few volume percent solid loading. ${ }^{13)}$ Flexible silicone rubber molds were replicated from rigid silicon molds in accordance with the soft-lithography procedure. The contacted PDMS $\mu$-mold forms micro-channels as shown in Fig. 1A. The microchannels are filled with the ethanol suspension. Fig. 1B shows a cross sectional image of the PDMS $\mu$-mold as solvent is evaporating. This sedimentation step is different from the standard
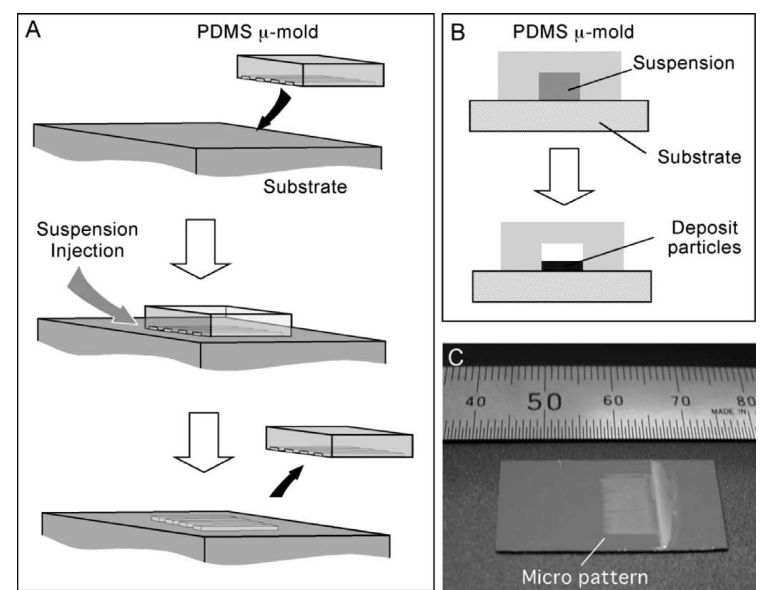

Fig. 1. Micro-pattern assembled with nano-particles by a micro molding in capillary method, MIMIC. A: A schematic flow chart of the MIMIC process, B: Sedimentation of particles in a PDMS $\mu$-mold and C: Micro pattern assembled with $\mathrm{SnO}_{2}$ nano-powders on a $\mathrm{Si}$ wafer. 
MIMIC techniques. After evaporation and solidification, the PDMS $\mu$-mold is peeled off the substrate. Figure $1 \mathrm{C}$ shows a micro pattern of tin oxide nano-powders formed on a silicon wafer.

Recently, there have been research efforts that focus on the miniaturization and the integration of the ceramic micro gas sensors. ${ }^{14), 15)}$ Assembling microstructures using nano-powders is one promising approach for processing ceramic gas sensors. In recent research involving semiconductor ceramics it has been shown that, by using structural control at the nanometer scale, ${ }^{16), 17)}$ the sensitivity of the gas sensor is increased and response time is improved. ${ }^{18)-20)}$ Heule and Gauckler first reported using the MIMIC process to assemble micro-sensors with nano-powders. ${ }^{15)}$ In this paper, we illustrate the application of a modified MIMIC method with a low load suspension of $\mathrm{SnO}_{2}$ nano-powders ${ }^{13)}$ to fabricate patterned micro gas sensors for detecting ethanol vapor.

\section{Experiment}

A commercially available ultra-fine tin oxide nano-powder (Nano Tek) was obtained from Kanto Chemical Co., Inc. The average powder particle diameter was $26 \mathrm{~nm}$. The nano-powder was dispersed in dehydrated ethanol (Kanto Chemical Co., Inc.) by supersonic vibration for $10 \mathrm{~min}$. An ethanol suspension of 2vol\% particulate concentration $(2.0 \mathrm{~g} / 15 \mathrm{ml})$ was prepared.

The PDMS micro-molds were constructed using a Polydimethylsiloxane elastomer kit (Sylgard 184, Dow Corning Inc., Midland, MI, USA) by replicating from a master mold made from a Si wafer. The master mold was fabricated on N-type [100] silicon wafers by a standard photolithography technique. The base to curing agent mixing ratio from the PDMS elastomer kit was 10:1. The mixture was poured over the master mold and degassed in a vacuum chamber for $30 \mathrm{~min}$. After curing the mold at $60^{\circ} \mathrm{C}$ for more than $3 \mathrm{~h}$, the PDMS $\mu$-mold was peeled from the master mold.

The channels of this PDMS $\mu$-mold form a set of parallel lines with each channel measuring $1.5 \mathrm{~cm}$ in length, $23 \mu \mathrm{m}$ in width and $3.5 \mu \mathrm{m}$ in depth. Figure $1 \mathrm{~A}$ shows the pattern of parallel PDMS lines attached to the upper surface of a substrate. A silicon wafer coated with a $1 \mu \mathrm{m}$ thick oxide layer (Furuya Metal Co., Ltd.) is used as a nonconductive substrate. To make the surface hydrophilic, the substrate was plasma-treated a priori. The drops of suspension were placed at the entrances of the channels of the PDMS mold, in increments of $10 \mu \mathrm{l}$ until a pool formed in front of the entrance. After the channels were filled and the interior dried, the suspension remaining at the entrance was wiped off and the PDMS mold was carefully peeled away. The

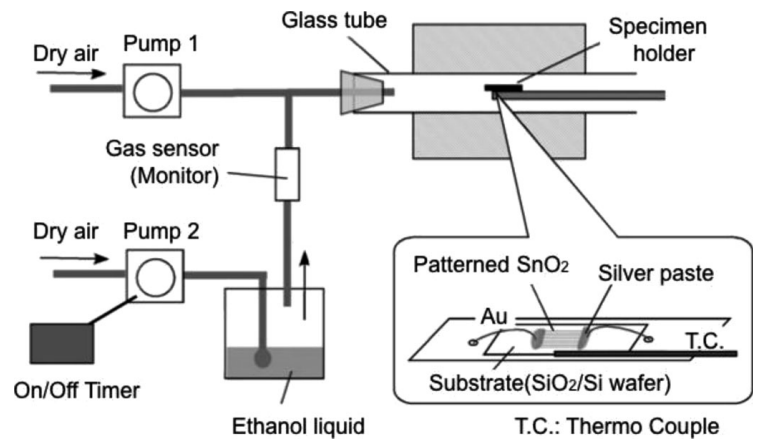

Fig. 2. A diagram of the property measurements made by the gas sensing system and electric tube furnace. silicon wafer was then patterned with $\mathrm{SnO}_{2}$ nano-powders and heated to $300^{\circ} \mathrm{C}$ for $5 \mathrm{~h}$ in air.

Microstructural images of the patterned nano-powders were taken with an optical microscope (Optiphoto II, Nikon Co.) and a field emission scanning electron microscope (S-5500 Hitachi, Ltd.). The thicknesses of the patterned nano-powders on the Si wafer were measured with a scanning probe microscope (SPI3700 Seiko Instrument Inc.). The performance of the patterned $\mathrm{SnO}_{2}$ gas sensor was assessed using the experimental system shown in Fig. 2. The ethanol vapor was generated by bubbling dry air (fed by Pump \#2) through liquid ethanol at a flow rate of $300 \mathrm{cc} / \mathrm{min}$. The generation of ethanol vapor was cycled using 10-min-on/30-min-off repetitions. The ethanol gas was mixed with dry air (fed by Pump \#1) at a flow rate of $300 \mathrm{cc} /$ min and then fed into the glass tube containing the specimen in a small electric furnace. A monitor gas sensor (TGS2620, Figaro Engineering, Inc.) was used to verify the production of the ethanol gas.

\section{Results and discussion}

Figure 1C shows a pattern of parallel lines fabricated on a silicon wafer substrate with a $1 \mu \mathrm{m}$ thick oxide layer. The pattern consists of more than 100 lines and the average line length is over $5 \mathrm{~mm}$. In our modified MIMIC process, we used suspensions of low solid loading, a requirement because of their low viscosity, and we used nano-sized powders to diminish the effect of the particles on capillary flow. In addition, ethanol has an affinity for the PDMS elastomer that forms the flexible molds. The procedure consists of only one instance of fluid deposition in front of the mold. Figure 3A shows an optical microscope image of the structure in which a clear line and space are observed. The width and the pitch distance of each line are approximately $23 \mu \mathrm{m}$ and $50 \mu \mathrm{m}$, respectively. Figure 3B shows an SEM image of the line. The surface of the line is almost flat. The lines have thicknesses that depend on the depth of mold channels. From the line profiling analysis of the SPM images, the average thickness of the line is found to be $1.2 \mu \mathrm{m}$. The thicknesses are nearly $1 / 3$ of the $3.5 \mu \mathrm{m}$ mold channel depth. Highresolution SEM images shown in Fig. 3C and Fig. 3D reveal the microstructure of the assembled nano-powder particle line in which the particle size varies non-uniformly from sizes smaller than $10 \mathrm{~nm}$ to larger than $50 \mathrm{~nm}$. The interior of the line is not densely packed with nano-powders, unlike a sintered ceramic.
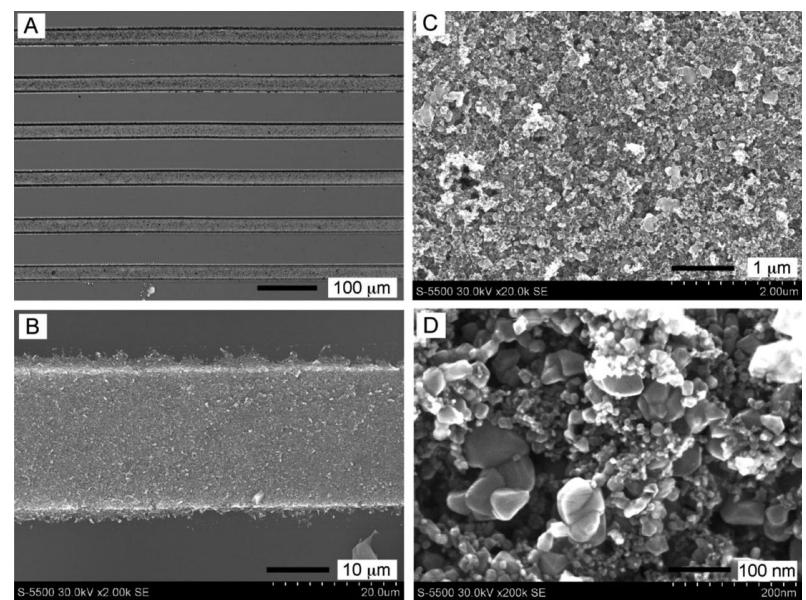

Fig. 3. Microstructure of patterned $\mathrm{SnO}_{2}$ nano-powders. A: Optical microscope image, B, C and D: High-resolution SEM images. 
Thus, such a microstructure has moderate strength and its porosity is useful for high sensitivity and high response gas-sensor applications.

Figure 4 shows an optical microscope image of $\mu$-gas sensor and its sensing property. Figure 4A shows six $\mathrm{SnO}_{2}$ powder micro-lines patterned between two Ag electrodes. A small amount of silver paste was used as an electrode. The precise electrode formation was accomplished by precision handwork under a magnifying glass. The Ag paste spreading process restricts the size of the gas sensor. The electrode formed consisted of six lines in this experiment. The average line length, line width and line height are $611 \mu \mathrm{m}, 23 \mu \mathrm{m}$ and $1.2 \mu \mathrm{m}$, respectively. Figure 4B shows the change in resistance of the $\mathrm{SnO}_{2} \mu$-gas sensor. The ethanol vapor passed for $10 \mathrm{~min}$ and then stopped for $30 \mathrm{~min}$ and the cycle was repeated as done in the case of the device shown in Fig. 2. The ethanol vapor flow was monitored using a commercially available Figaro TGS2620 gas-sensor. The flow was
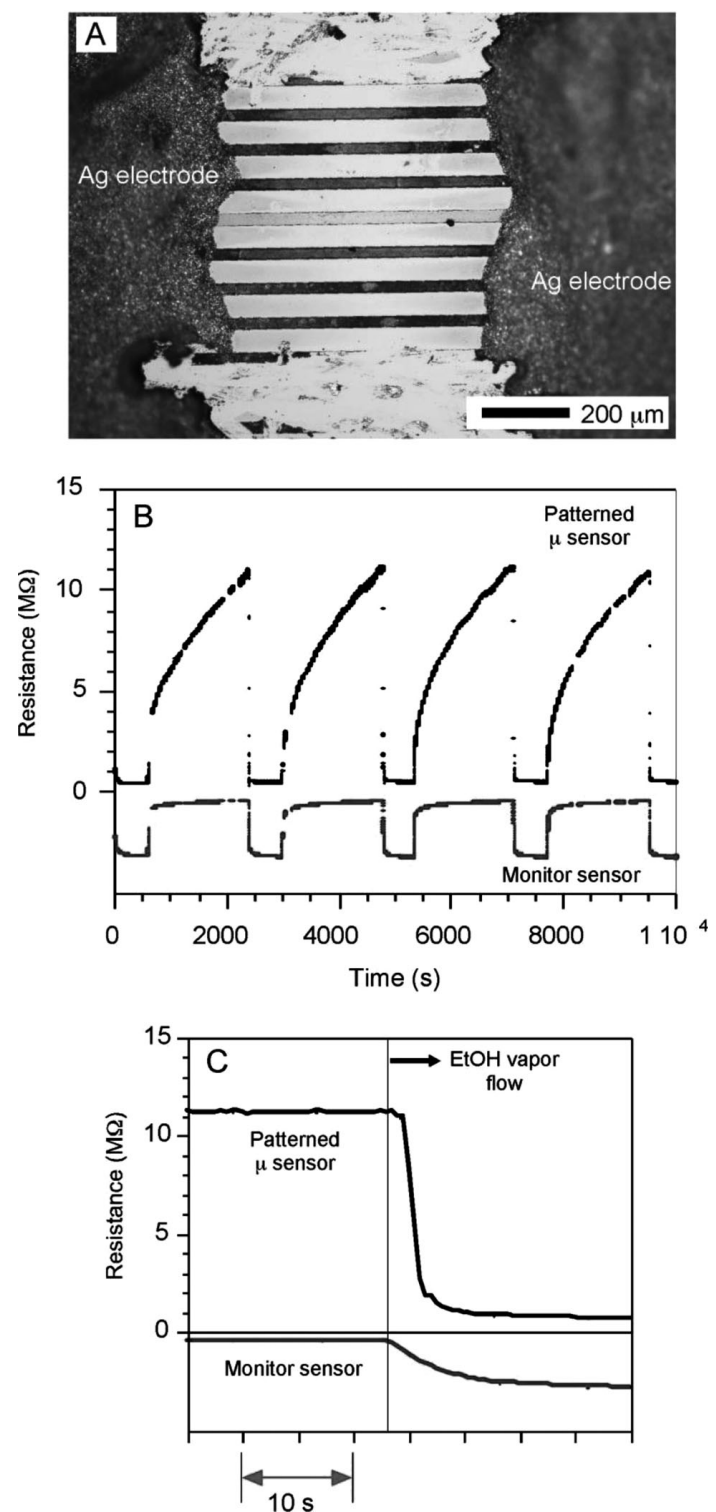

Fig. 4. $\mathrm{SnO}_{2}$ patterned micro gas sensor. A: Optical microscope image between two electrodes. Six lines were fixed with silver paste. B: Interval change of electric resistance by ethanol vapor at $450^{\circ} \mathrm{C}$. C: Comparison of a micro gas sensor and a commercial available monitor sensor. monitored in-situ before it was mixed with dry air from pump \#1 as shown in Fig. 2. The resistivity, $\rho(\Omega \mathrm{m})$ is calculated from the expression, $\rho=\mathrm{RA} / 6 \mathrm{~L}$, where R is the electric resistance $(\Omega)$, $\mathrm{L}$ is the line length $(\mathrm{m})$ and $\mathrm{A}$ is the sectional area $\left(\mathrm{m}^{2}\right)$. The electrical resistance in the dry state and in the ethanol vapor was found to be $11.13 \mathrm{M} \Omega$ and $0.574 \mathrm{M} \Omega$, respectively. The measured resistivity of the line assembled with the $\mathrm{SnO}_{2}$ nanopowders in dry air $\rho_{\text {air }}$ is $8.4 \cdot 10^{-2} \Omega \mathrm{m}$ and in ethanol vapor $\rho_{\mathrm{EtOH}}$ is $4.3 \cdot 10^{-3} \Omega \mathrm{m}$. These values compare reasonably well with the results of Tischner et al. ${ }^{14)}$ The sensor resistance ratio as a measurement of the magnitude of gas response, $\mathrm{SR}=\rho_{\text {air }} / \rho_{\mathrm{EtOH}}$, is found to be 19.5 at $450^{\circ} \mathrm{C}$, compared to the reference value of the gas sensor $\mathrm{SR}=8.5$ measured outside the glass tube. The patterned micro gas sensor has a large enough SR value to easily detect ethanol vapor without error.

Figure 4C shows the change in the resistance of the gas sensor when the ethanol vapor flows in. The upper plot is a time change of the resistance of the gas vapour of the micro gas sensor at $450^{\circ} \mathrm{C}$. The response time for the ethanol vapor is $1.9 \mathrm{~s}$. In contrast, the response time of the monitor gas sensor is $3.9 \mathrm{~s}$, as shown in the lower graph in Fig. 4C. The response speed of the micro gas sensor is a large enough value so as to be comparable in performance to commercially available gas sensors. It should be noted that the delay in the time marking at the beginning of the resistance change is due to the difference in the location of the measurement of the ethanol gas as shown in Fig. 2.

In this paper, we have demonstrated that micro lines assembled with tin oxide nano-powders act as an ethanol vapor sensor. In order to safeguard our society from harmful toxins, the electric nose that can be used to detect various gases is a very important research product. Our modified MIMIC technique that uses a colloidal ceramic suspension is a useful and cost-effective processing technique to integrate multiple gas sensors. Finally, if each line of the PDMS $\mu$-mold can be filled with a different material for gas sensing, a multiple chemical gas sensor can be fabricated at low cost and with low energy consumption. Furthermore, such a device can be used in the field for many energy, environmental and health and safety applications.

\section{Conclusion}

A micro gas sensor, assembled using $\mathrm{SnO}_{2}$ nano-powders in a 2 vol\% ethanol suspensions by capillary flow in a micro molding process, has been developed and demonstrated. The patterned $\mathrm{SnO}_{2}$ nano powders act as a gas sensor for ethanol vapor. The sensing resistance ratio is 19.5 and the gas response time is $1.9 \mathrm{~s}$ at $450^{\circ} \mathrm{C}$. The response speed of the micro gas sensor is comparable to gas sensors currently available on the market. We have demonstrated that our modified MIMIC method enables the fabrication of a micro gas sensor without special equipment.

Acknowledgement This work was financially supported by the Hosokawa Powder Technology Foundation (\#03106) and by a grantin-aid (KIBAN B: \#16350117) for scientific research from the Ministry of Education, Science and Culture of Japan. HF thankfully acknowledges Prof. Younan Xia, of Washington University in St. Louis, for support in sharing his soft lithography resources and Mr. Shinichi Hara, of NIMS nano-foundry station, for his support in the fabrication of the silicon master molds. HF also thanks Ms. Jyunko Imasu and Ms. Naomi Tawarayama for their technical assistance. We gratefully acknowledge the materials analysis station of NIMS for their technical supported in the taking of the high-resolution SEM images. 


\section{References}

1) M. Heule, J. Schell and L. J. Gauckler, J. Am. Ceram. Soc., 86, 407-412 (2003).

2) X. M. Zhao, J. Mater. Chem., 7, 1069-1074 (1997).

3) O. F. Göbel, M. Nedelcu and U. Steiner, Adv. Funct. Mater., 17, 1131-1136 (2007).

4) E. Kim, Y. Xia and G. M. Whitesides, J. Am. Chem. Soc., 118, 5722-5731 (1996).

5) Y. Xia and G. M. Whitesides, Angew. Chem. Int. Ed., 37, 550575 (1998).

6) Y. Xia and G. M. Whitesides, Ann. Rev. Mater. Sci., 28, 153184 (1998).

7) W. S. Beh, Y. Xia and D. Qin, J. Mater. Res., 14, 3995-4003 (1999).

8) C. R. Martin and I. A. Aksay, J. Phys. Chem. B, 107, 42614268 (2003).

9) S. J. Ahn and J. Moon, J. Ceram. Soc. Japan, 112, S156-S158 (2004).

10) S. J. Ahn and J. Moon, J. Am. Ceram. Soc., 88, 1171-1174
(2005).

11) S. J. Ahn, J. H. Min, J. Kim and J. Moon, J. Am. Ceram. Soc., 91, 2143-2149 (2008).

12) J. Imasu, H. Fudouzi and Y. Sakka, J. Ceram. Soc. Japan, 114, 725-728 (2006).

13) J. Imasu, H. Fudouzi and Y. Sakka, Ceram. Trans., 198, 251256 (2007).

14) A. Tischner, T. Maier, C. Stepper and A. Köck, Sens. Actuat. $B, 134,796-802$ (2008).

15) M. Heule and L. J. Gauckler, Sens. Actuat. B, 93, 100-106 (2003).

16) Y. Shimizu and M. Egashira, MRS Bulletin, 24, 18-24 (1999).

17) N. Yamazoe, Sens. Actuat. B, 5, 7-19 (1991).

18) W. Gopel and K. D. Schierbaum, Sens. Actuat. B, 26, 1-12 (1995).

19) G. Martinelli, M. C. Carotta, E. Traversa and G. Ghiotti, $M R S$ Bulletin, 24, 30-36 (1999).

20) G. Zhang and M. L. Liu, Sens. Actuat. B, 69, 144-152 (2000). 